\title{
Comparison of Adaptability of Three Different Root Canal Obturation Materials
}

\author{
${ }^{1} \mathrm{~K}$ Kranthi Raja, ${ }^{2}$ Afaf Al-Haddad, ${ }^{3}$ Padmini Hari, ${ }^{4}$ Kiran P Singbal
}

\begin{abstract}
Objective: The aim was to evaluate and compare the adaptability of the three obturation materials: gutta-percha, GuttaFlow, and Soft-Core to the root canal walls.

Materials and methods: Totally 30 mandibular premolar teeth were selected. The teeth were standardized and prepared with ProTaper rotary files. The teeth were randomly and equally divided into three groups-gutta-percha group: obturated with gutta-percha and $\mathrm{AH}$ Plus sealer using lateral compaction technique; Soft-Core group: obturated using Soft-Core system; and GuttaFlow: obturated with GuttaFlow. The roots of the teeth were sectioned at three levels (apical, middle, and coronal) and were observed under a stereomicroscope at $40 \times$ magnification. Area of voids (AVs), frequency of voids, and location of the voids were analyzed using image analysis software.
\end{abstract}

Results: The lowest mean of AV was obtained by GuttaFlow $1.25 \% \pm 1.93$, followed by gutta-percha $1.33 \% \pm 2.16$, and SoftCore $1.74 \% \pm 2.23$. Statistical analysis showed no significant difference among the three groups and the levels of the root. The frequency of voids in the coronal and the middle levels was more than the apical for all groups. The highest frequency of voids was detected in Soft-Core, followed by GuttaFlow and gutta-percha respectively. The voids were located in the interphase between sealer and obturation material as well as sealer and root canal walls in the gutta-percha and Soft-Core groups, whereas it was almost confined to the core for GuttaFlow group.

Conclusion: GuttaFlow has a better adaptability to the walls compared with Soft-Core system and laterally compacted gutta-percha with AH Plus sealer.

Clinical significance: GuttaFlow is an appropriate obturation material to enhance endodontic treatment.

Keywords: Apical seal, GuttaFlow, Obturation of root canal, Sealing ability, Soft-Core.

How to cite this article: Raja KK, Al-Haddad A, Hari P, Singbal KP. Comparison of Adaptability of Three Different Root Canal Obturation Materials. World J Dent 2017;8(6):452-456.

Source of support: Nil

Conflict of interest: None

\footnotetext{
${ }^{1,4}$ Department of Conservative Dentistry, Faculty of Dentistry Mahsa University, Selangor, Malaysia

${ }^{2}$ Department of Dental Materials and Equipment, Faculty of Dentistry, Mahsa University, Selangor, Malaysia

${ }^{3}$ Department of Periodontology, Faculty of Dentistry, Mahsa University, Selangor, Malaysia

Corresponding Author: K Kranthi Raja, Department of Conservative Dentistry, Faculty of Dentistry, Mahsa University Selangor, Malaysia, Phone: +0060166590480, e-mail: kranthi@ mahsa.edu.my
}

\section{INTRODUCTION}

Three-dimensional obturation of the root canal system promotes periapical healing and prevents disease progression. The hermetic seal created at the apical dentinocemental junction will determine the success of obturation. $^{1}$

Gutta-percha, until today, has been accepted as the most common material for root canal filling, as it is not toxic and will not irritate periapical tissues. ${ }^{2,3}$ Guttapercha has a disadvantage of lack of bonding to canal dentin. ${ }^{4}$ Therefore, it is usually combined with root sealer to fulfill the obturation objective. AH Plus (Dentsply De Trey, Konstanz, Germany) is a resin-based sealer most commonly used with gutta-percha in vertical or lateral compactions. AH Plus has an adequate long-term dimensional stability, but its sealing ability remains questionable as it does not bind to gutta-percha. ${ }^{5}$

Cold lateral compaction has been used frequently based on comparison of new root canal filling techniques and materials. ${ }^{4}$ In the cold lateral condensation technique, spaces between the gutta-percha cones can result due to inadequate condensation pressure or a mismatch between the tapers of the spreader, gutta-percha cone, and the canal. Therefore, many materials and techniques have been developed with an objective to offer a higher sealing ability. ${ }^{6}$

In 1978, Johnson had introduced Soft-Core technique (Aps, Copenhagen, Denmark), a thermoplasticized obturation technique, which involved the use of a metal carrier coated with a layer of gutta-percha that was heated to permit thermoplasticized canal obturation. ${ }^{4,7}$ Soft-Core offers advantages, such as a reduction in chairside time and rapid setting of the gutta-percha. ${ }^{8}$

GuttaFlow (Coltene/Whaledent, Altstatten, Switzerland) is cold fluid obturation system that combines sealer and gutta-percha. GuttaFlow includes particulate gutta-percha in a polydimethyl siloxane base, which has good adaptability because of the increased flowability and better seal. ${ }^{9}$ GuttaFlow has got good fluidity for providing a thin film of sealer. Its antibacterial, insolubility, biocompatibility, and postsetting expansion properties, as claimed by the manufacturer, are promising as an obturating material.

Various techniques have been used in previous studies to evaluate adaptation of root canal filling materials to the root canal walls, such as the dye penetration and 
radioisotope techniques, penetration of microorganisms, and electrochemical technique. ${ }^{10,11}$ Each of these methods has its own advantages and disadvantages. Nevertheless, assessing cross-sections of the roots under a stereomicroscope was demonstrated to provide a three-dimensional view of the surface to be examined, which aids in eliminating human errors in the interpretation of the parameters. ${ }^{7}$

Thus, the aim of the current study was to evaluate and compare the sealing ability and adaptation of GuttaFlow single-cone technique in comparison with gutta-percha/AH Plus sealer using lateral condensation technique and Softcore system/AH Plus sealer under a stereomicroscope.

\section{MATERIALS AND METHODS}

\section{Sample Selection}

Totally, 30 single-rooted, human mandibular premolar teeth of similar length extracted in the past 3 months for periodontal reasons were selected. All teeth were cleaned to remove the extraneous tissue and calculus. The teeth were visually examined and verified to have single canal, a closed apex, and no curvatures. The crown of each tooth was removed at the level of the cementoenamel junction with a diamond disk to obtain root segments. Patency of the apical foramina was standardized by inserting a size 15 K-file (Prime Dental Products Pvt Ltd), so that the tip was just visible. Individual working length (WL) was calculated $0.5 \mathrm{~mm}$ short of this position. Teeth with diameters larger than size 15 , teeth with sclerotic canals, or teeth with an altered apex were excluded from the study.

\section{Canal Preparation}

Samples were prepared with ProTaper nickel-titanium instruments (Dentsply Maillefer) according to the manufacturer's instructions. A 64:1 Gear reduction handpiece (Dentsply Maillefer) powered with a torque-controlled electric stepper motor was used with a consistent speed of $300 \mathrm{rpm}$. Rotary instruments were used in a crown-down method in a sequential order till F3. All the canals were irrigated initially with $3 \mathrm{~mL}$ of $5 \%$ sodium hypochlorite, followed by $1 \mathrm{~mL}$ of $5 \%$ sodium hypochlorite between each instrument. Each prepared canal was then rinsed with $17 \%$ ethylenediaminetetraacetic acid (EDTA). The canals were then dried with paper points. All irrigation procedures were delivered with a 27-gauge needle (Dispo Van, HMD Ltd). After the complete instrumentation and irrigation, each canal was dried with sterile paper points.

\section{Obturation of the Root Canal}

The teeth were randomly divided into three groups $(n=10)$ and obturated as follows:
- Group I: Specimens obturated with gutta-percha and $\mathrm{AH}$ Plus sealer using lateral compaction technique. Briefly, a master gutta-percha cone size 30/0.02 was introduced into the canal to the WL until a tug-back is obtained. The AH Plus sealer was mixed according to manufacturer's instructions. The sealer was applied along the entire length of the canal wall using paper points. The master gutta-percha cone was lightly coated with sealer and inserted into the canal. Lateral compaction was then accomplished using a fine-sized finger spreader (Dentsply Maillefer) and fine-sized accessory gutta-percha points (Dentsply Maillefer). Compaction continued until the spreader could not be inserted more than $2 \mathrm{~mm}$ into the canal. The excess gutta-percha was removed using a heated instrument, and the guttapercha was vertically compacted using hand plugger.

- Group II: Specimens obturated using Soft-Core system with AH Plus sealer. The size of the Soft-Core system (Aps, Copenhagen, Denmark) obturator was selected using the International Organization for Standardization verifier. The AH Plus sealer was introduced into the canal. The obturator was then placed in the Soft-Core oven. When the oven indicated the obturator was ready, it was removed from one of the slots in the top of oven and inserted until the WL. The handle and insertion pin were removed by twisting motion. The excess plastic core material was removed with a small inverted cone bur. The gutta-percha was then compacted vertically with hand plugger.

- Group III: Specimens obturated with GuttaFlow (Coltene/Whaledent, Altstatten, Switzerland). Size 30/0.02 master gutta-percha cone was selected and introduced into the canal to the WL until a tug-back was obtained. GuttaFlow capsules were vibrated using an amalgamator for 30 seconds according to the manufacturer's instructions. The tip of the GuttaFlow device was inserted into the root canal $3 \mathrm{~mm}$ short of the WL, and the filling material was introduced until the flow of the material inside the canal could be observed ensuring that the material does not extrude through the apical foramen. A fresh mix was obtained on a glass slab by pressing the mixing pistol. The gutta-percha master cone was coated with GuttaFlow sealer and inserted to the WL. By pressing the master cone laterally, the tip of the device was inserted again into the canal to seal the space left with backfilling technique. The excess material was removed with an excavator. The gutta-percha was then compacted vertically with a hand plugger.

For all groups, radiographs were taken at buccal and mesial aspects to assess the quality of root canal filling that should be dense without voids and extended within 1 $\mathrm{mm}$ from the root end. All roots were restored with IRM (Dentsply International, Caulk, Milford, Delaware, USA). 
Table 1: Mean percentage of $\mathrm{AV}$ of three obturation materials

\begin{tabular}{lllll}
\hline Material & Coronal mean (SD) & Middle mean (SD) & Apical mean (SD) & Total mean (SD) \\
\hline Gutta-percha with lateral condensation & $1.17(1.75)$ & $1.53(2.00)$ & $1.27(2.8)$ & $1.330(2.16)$ \\
Soft-Core & $2.41(2.64)$ & $1.64(1.84)$ & $0.7(1.18)$ & $1.744(2.23)$ \\
GuttaFlow & $1.88(2.27)$ & $0.46(0.79)$ & $1.14(1.96)$ & $1.246(1.93)$ \\
p-value & $0.420^{*}$ & $0.295^{*}$ & $0.936^{*}$ & $0.427^{*}$ \\
\hline
\end{tabular}

*Statistically not significant at $p \geq 0.05$; SD: Standard deviation

All specimens were kept in an incubator at $37^{\circ} \mathrm{C}$ in $100 \%$ humidity for 1 week to allow sealers to set.

\section{Sectioning the Samples}

Each root was sectioned at three levels according to the individual root length as follows: coronal (at the middle of coronal-third), middle (at the middle of middle-third), and apical (at the middle of apical third). Sectioning was done using Isomet saw (Buehler, USA) under water coolant. The thickness of the sections was $2 \mathrm{~mm}$. The samples were observed under stereomicroscope (Olympus SZ 650, Japan) at $\times 40$ magnification. Area of voids and number of sections with voids, i.e., frequency of voids and location of voids (either in the filling core or along the root canal wall) were recorded using Image analyzer software $\left(C e l l^{\wedge} \mathrm{D}\right.$, version $2.4 .112-240608$, Olympus). Area of the entire root canal and that of voids were outlined and measured. The AV was calculated as the percentage of voids occupying the cross-section.

\section{Statistical Analysis}

Kruskal-Wallis test was used to analyze the variance among different obturating materials for each level of the canal and total levels. Analysis of variance (ANOVA) repeated measures were used to analyze the variance among root canal levels for each obturation material. The differences in the parameters tested were considered statistically significant at a $\mathrm{p} \geq 0.05$.

\section{RESULTS}

The mean percentage of $\mathrm{AV}$ of three groups is shown in Table 1. Kruskal-Wallis showed no significant difference in the AV among the three different materials, gutta-percha, GuttaFlow, and Soft-Core, regardless of the level of the root canal $(p=0.427)$ as well as in each level of the root canal: coronal level $(\mathrm{p}=0.420)$, middle level $(\mathrm{p}=0.295)$, and apical level $(\mathrm{p}=0.936)$.

The ANOVA repeated measures analysis showed that there was no significant difference in AV between the coronal, the middle, and the apical levels for each obturation material. $\mathrm{F}(2,18)=0.641, \mathrm{p}=0.538$ for gutta-percha group, $\mathrm{F}(2,18)=1.96, \mathrm{p}=0.169$ for Soft-Core group, and $\mathrm{F}(2,18)=0.165, \mathrm{p}=0.849$ for GuttaFlow group.

The frequency of voids in the coronal and middle sections was more compared with the apical sections
Table 2: Frequency of voids in the coronal, middle, and apical levels of the root canal

\begin{tabular}{lllll}
\hline Material & $\begin{array}{l}\text { Coronal } \\
(n=10)\end{array}$ & $\begin{array}{l}\text { Middle } \\
(n=10)\end{array}$ & $\begin{array}{l}\text { Apical } \\
(n=10)\end{array}$ & $\begin{array}{l}\text { Total } \\
(n=30)\end{array}$ \\
\hline $\begin{array}{l}\text { Gutta-percha with lateral } \\
\text { condensation }\end{array}$ & 4 & 5 & 2 & 11 \\
$\begin{array}{l}\text { Soft-Core } \\
\text { GuttaFlow }\end{array}$ & 7 & 7 & 3 & 17 \\
\hline
\end{tabular}

in all the three groups. The highest number of sections with voids was seen in the Soft-Core group, followed by GuttaFlow and gutta-percha respectively, as shown in Table 2.

The voids were more frequently found in the interphase between sealer and obturation material as well as sealer and root canal walls in the gutta-percha and Soft-Core groups, whereas it was almost confined to core with GuttaFlow group as shown in Figure 1.

\section{DISCUSSION}

The aim of endodontic treatment is to prevent the microleakage along the root canal. The success of threedimensional obturation of root canal depends on the type of the obturating materials. ${ }^{12}$ The presence or absence of voids is a very important issue associated with the assessment of canal filling quality.

Selection of the teeth and their assignment into different study groups were done using stratified randomization, and preparation of the canals was performed by one operator using standard techniques to minimize anatomical variation, reduce the variability all the canals, and allow standardization. Sectioning of teeth was not performed at predetermined distances from apex, but done according to individual root length. This was done to obtain comparable information at specific root levels. ${ }^{9}$

The removal of smear layer may be considered as an essential in the process of successful root canal treatment. It is well known that root canal filling materials penetrate better into dentinal tubules in the absence of the smear layer. For this reason, $17 \%$ EDTA solution and $5 \%$ sodium hypochlorite were used to remove the smear layer and facilitate the penetration and adaptation of root canal filling materials in the previous study.,13

In this study, the Soft-Core group revealed the highest number of sections with voids, followed by GuttaFlow and laterally condensed gutta-percha. In Soft-Core, stripping of the gutta-percha from the solid core-carrier has been 

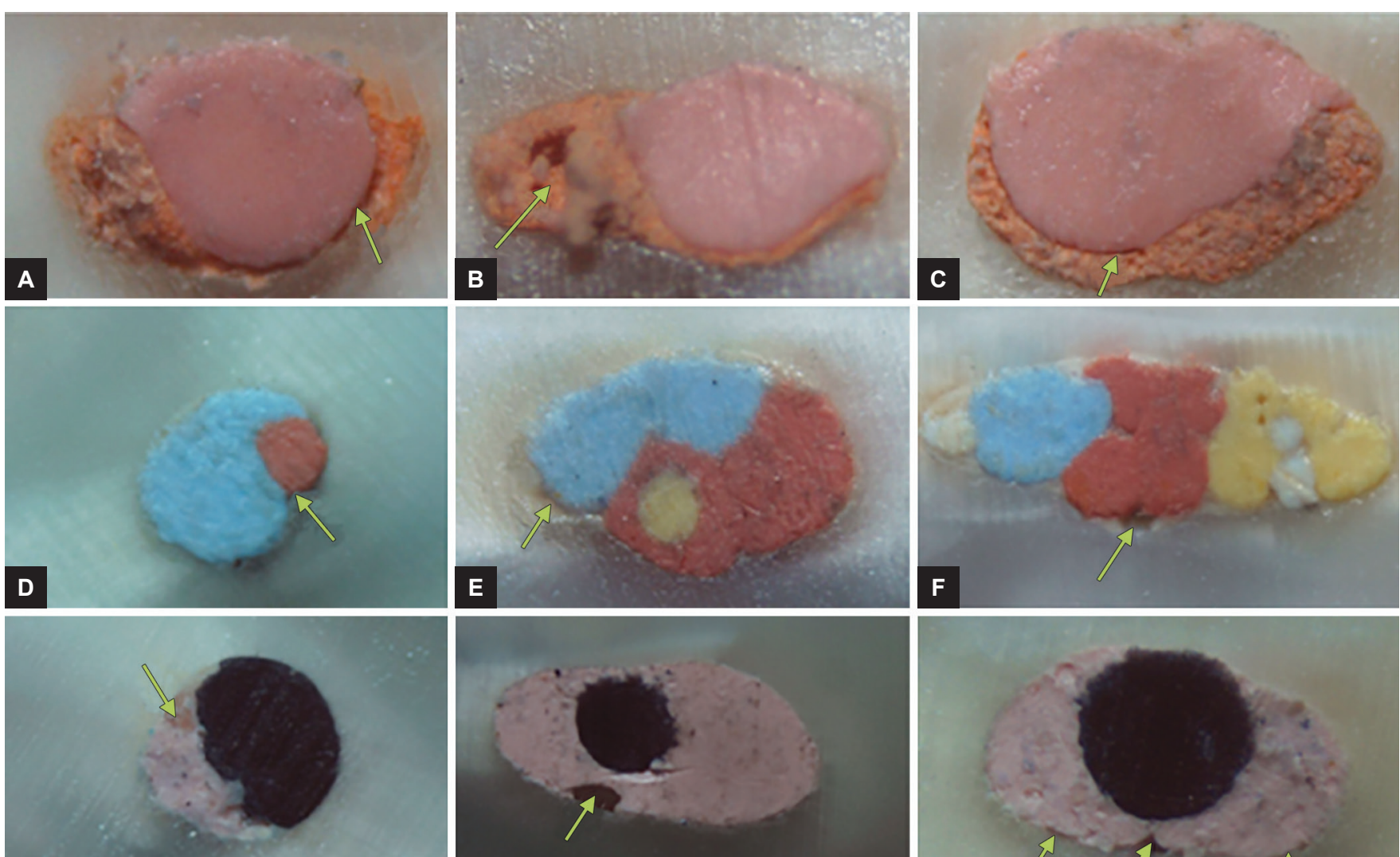

G
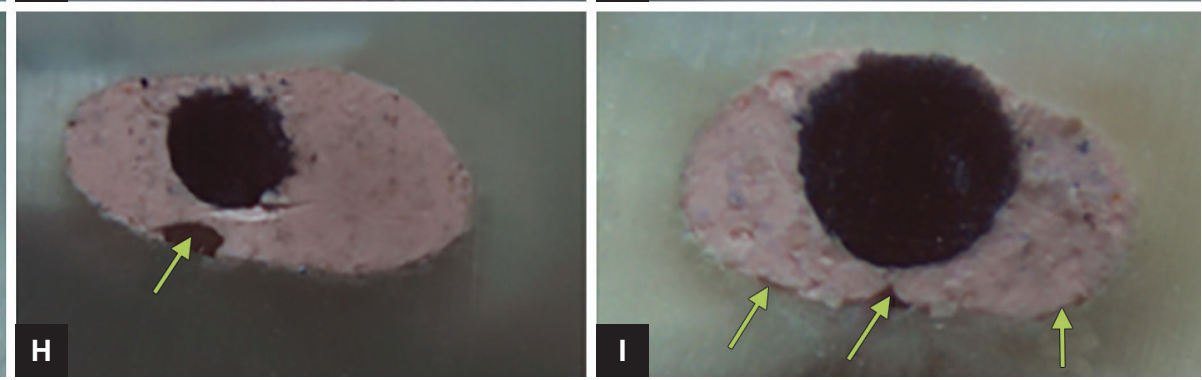

Figs 1A to I: Representative stereomicroscopic images of three obturation materials at apical, middle, and coronal thirds of root canals. GuttaFlow group: (A) Apical, (B) middle, and (C) coronal thirds. Gutta-percha groups: (D) apical, (E) middle, and (F) coronal thirds. Soft-Core group: $(\mathrm{G})$ apical, $(\mathrm{H})$ middle, and (I) coronal thirds. The voids (arrowed) were located in the interphase between sealer and obturation material as well as sealer and root canal walls in the gutta-percha and Soft-Core groups, whereas it was almost confined to the core for the GuttaFlow group

demonstrated in a previous study. ${ }^{14}$ This phenomenon will allow the carrier to be in direct contact with the canal walls, which results in more voids due to the poor adaptation of solid carrier to the irregularities in the dentinal wall.

The sections with voids were more in the middle-third of both laterally condensed gutta-percha and Soft-Core groups. This could be attributed to narrow oval canals of premolars in the middle-thirds. It was demonstrated that oval canals had uninstrumented area due to inaccessibility of files and subsequently may lead to incomplete obturation. ${ }^{14}$ The least number of sections with voids in all the groups was seen in the apical third. This could be referred to the round shape of apical third of the canals that increases the matching fit of the master point and subsequently the adaptation of filling material. ${ }^{15}$

The frequency of voids with GuttaFlow group was more than gutta-percha group; however, size of voids in GuttaFlow was remarkably small, thus resulting in the least percentage of $\mathrm{AVs}$ compared with the other two groups. ${ }^{9}$ Similar studies conducted found that microleakage of GuttaFlow using a single-cone technique is similar to that of gutta-percha using lateral condensation and less than that of gutta-percha using vertical condensation. ${ }^{16}$
GuttaFlow, when used with a single gutta-percha master cone, created an apical seal that is equivalent to that produced with gutta-percha/AH Plus sealer using warm vertical compaction. ${ }^{17}$ When lateral compaction and single-cone obturation techniques using GuttaFlow were compared with AH Plus as sealer, they showed a greater apical and coronal sealing ability over time. ${ }^{18}$

Contrary to the current study, GuttaFlow exhibited maximum apical microleakage in root canals compared with resilon, thermafil, and lateral condensation in a study conducted by Punia et al. ${ }^{19}$

In accordance with the present study, studies comparing cold lateral condensation and Soft-Core obturation technique showed no significant difference in the adaptation to root canal walls. ${ }^{8}$ In another similar study using dye penetration technique, Softcore resulted in the highest mean number of voids compared with cold lateral condensation and hybrid gutta-percha condensation technique. They have also reported that the mean apical leakage for the Softcore technique was at least twice as extensive as for the two other gutta-percha obturation techniques. ${ }^{20}$

The present study confirms that none of the obturating materials provided a gap-free or a void-free root canal 
filling. ${ }^{14}$ The size of individual voids in GuttaFlow was small and mostly they were confined to the core of the material, whereas the size of the voids in other two groups was relatively larger and the voids were seen at the interphase. GuttaFlow group showed up as the most homogeneous sealer with no defects compared with gutta-percha lateral condensation and Soft-Core techniques. The presence of fine-sized gutta-percha particles (nanoparticles $<30 \mu$ ) further bestows increased flow ability to GuttaFlow, resulting in better coating capacity and adaptation to root canal walls, as well as into dentinal tubules. ${ }^{21}$ This can be attributed to the manufacture processing and highly viscous composition resulting in the homogeneous distribution of the sealer without any voids. ${ }^{9}$

Within the confines of this study, despite the presence of small voids within the core of the material, GuttaFlow exhibited better adaptability compared with gutta-percha lateral condensation and Soft-Core materials. This might be due to a $0.2 \%$ expansion as claimed by the manufacturer combined with the close adaptation of the guttapercha cone against the walls. ${ }^{9}$

\section{CONCLUSION}

GuttaFlow has a better adaptability to the walls compared with the Soft-Core system and laterally compacted guttapercha with AH Plus sealer.

\section{REFERENCES}

1. Schilder H. Filling root canal in three dimensions. Dent Clin North Am 1967 Nov:723-744.

2. Tunga U, Bodrumlu E. Assessment of the sealing ability of a new root canal obturation material. J Endod 2006 Sep;32(9):876-878.

3. Stratton RK, Apicella MJ, Mines P. A fluid filtration comparison of gutta-percha versus resilon, a new soft resin endodontic obturation system. J Endod 2006 Jul;32(7):642-645.

4. De Moor RJ, Hommez GM. The long-term sealing ability of an epoxy resin root canal sealer used with five gutta percha obturation techniques. Int Endod J 2002 Mar;35(3):275-282.

5. Bouillaguet S, Shaw L, Barthelemy J, Krejci I, Wataha JC. Long-term sealing ability of pulp canal sealer, AH-plus, gutta flow and epiphany. Int Endod J 2008 Mar;41(3):219-226.

6. Shipper G, Trope M. In vitro microbial leakage of endodontically treated teeth using new and standard obturation techniques. J Endod 2004 Mar;30(3):154-158.

7. Gencoglu N. Comparison of 6 different gutta-percha techniques: thermafil, JS quick-fill, softcore, microseal, system B and lateral condensation. Oral Surg Oral Med Oral Pathol Oral Radiol Endod 2003 Jul;96(1):91-95.

8. Ozer SY, Aktener BO. Outcome of root canal treatment using soft-core and cold lateral compaction filling techniques: a randomized clinical trial. J Contemp Dent Pract 2009 Jan 1;10(1):74-81.

9. Elayouti A, Achleithner C, Löst C, Weiger R. Homogeneity and adaptation of a new gutta-percha paste to root canal walls. J Endod 2005 Sep;31(9):687-690.

10. Merces A, Carlos A, Camara A. Comparison of root canals obturated with pro taper gutta-percha master point using the active lateral condensation and the single cone techniques: a bacterial leakage study. Braz J Oral Sci 2011;10:37-41.

11. Bal AS, Hicks ML, Barnett F. Comparison of laterally condensed .06 and .02 tapered gutta-percha and sealer in vitro. J Endod 2001 Dec;27(12):786-788.

12. Patil P, Rathore VP, Hotkar C, Savgave SS, Raghavendra K, Ingale P. A comparison of apical sealing ability between guttaflow and $\mathrm{AH}$ plus: An in vitro study. J Int Soc Prev Community Dent 2016 Jul-Aug;6(4):377-382.

13. Gençoğlu N, Samani S, Günday M. Dentinal wall adaptation of thermoplasticized gutta-percha in the absence or presence of smear layer: a scanning electron microscopic study. J Endod 1993 Nov;19(11):558-562.

14. De Moor RJ, Martens LC. Apical microleakage after lateral condensation, hybrid gutta-percha condensation and softcore obturation: an in vitro evaluation. Endod Dent Traumatol 1999 Oct;15(5):239-243.

15. Wu MK, Wesselink PR. A primary observation on the preparation and obturation of oval canals. Int Endod J 2001 Mar;34(2):137-141.

16. Vertucci FJ. Root canal morphology of mandibular premolars. J Am Dent Assoc 1978 Jul;97(1):47-50.

17. Pitout E, Oberholzer TG. Leakage of teeth root-filled with guttaflow and a single GP cone compared to lateral condensation and warm vertical condensation. SADJ 2009 Apr; 64(3):104, 106-108.

18. Brackett MG, Martin R, Sword J, Oxford C, Rueggeberg FA, Tay FR, Pashley DH. Comparison of seal after obturation techniques using a polydimethylsiloxane-based root canal sealer. J Endod 2006 Dec;32(12):1188-1190.

19. Punia SK, Nadig P, Punia V. An in vitro assessment of apical microleakage in root canals obturated with gutta-flow, resilon, thermafil and lateral condensation: a stereomicroscopic study. J Conserv Dent 2011 Apr;14(2):173-177.

20. Rana M, Sandhu GK, Kaur T, Arif M, Galyan G. New selfcuring root canal filling material: Gutta flow 2. J Adv Med Dent Sci Res 2014;2:15-20.

21. Gernhardt CR, Krüger T, Bekes K, Schaller HG. Apical sealing ability of 2 epoxy resin-based sealers used with root canal obturation techniques based on warm gutta-percha compared to cold lateral condensation. Quintessence Int 2007 Mar;38(3):229-234. 